\title{
Estimated cumulative radiation dose from PET/CT in children with malignancies: a 5-year retrospective review
}

\author{
Soni C. Chawla • Noah Federman • Di Zhang • \\ Kristen Nagata $\cdot$ Soujanya Nuthakki $\cdot$ \\ Michael McNitt-Gray • M. Ines Boechat
}

Received: 1 October 2008 /Revised: 9 September 2009/Accepted: 1 October 2009/Published online: 5 December 2009

(C) The Author(s) 2009. This article is published with open access at Springerlink.com

\begin{abstract}
Background The increasing use of serial PET/CT scans in the management of pediatric malignancies raises the important consideration of radiation exposure in children. Objective To estimate the cumulative radiation dose from $\mathrm{PET} / \mathrm{CT}$ studies to children with malignancy and to compare with the data in literature.

Materials and methods Two hundred forty-eight clinical PET/CT studies performed on 78 patients $(50$ boys/28 girls, 1.3 to 18 years old from December 2002 to October 2007) were retrospectively reviewed under IRB approval. The whole-body effective dose (ED) estimates for each child were obtained by estimating the effective dose from each $\mathrm{PET} / \mathrm{CT}$ exam performed using the ImPACT Patient Dosimetry Calculator for CT and OLINDA for PET.

Results The average number of PET/CT studies was 3.2 per child (range: 1 to 14 studies). The average ED of an individual CT study was $20.3 \mathrm{mSv}$ (range: 2.7 to 54.2), of PET study was $4.6 \mathrm{mSv}$ (range: 0.4 to 7.7 ) and of PET/CT study was
\end{abstract}

S. C. Chawla $(\bowtie)$

Department of Radiology,

Olive View-UCLA Medical Center,

14445 Olive View Drive,

2 D115 Sylmar, CA, USA

e-mail: chawlasoni@gmail.com

N. Federman

Department of Pediatrics,

David Geffen School of Medicine at UCLA,

Los Angeles, CA, USA

D. Zhang

Department of Biomedical Physics,

David Geffen School of Medicine at UCLA,

Los Angeles, CA, USA
$24.8 \mathrm{mSv}$ (range: 6.2 to 60.7 ). The average cumulative radiation dose per patient from CT studies was $64.4 \mathrm{mSv}$ (range: 2.7 to 326), from PET studies was $14.5 \mathrm{mSv}$ (range: 2.8 to 73 ) and from PET/CT studies was $78.9 \mathrm{mSv}$ (range: 6.2 to 399$)$.

Conclusion The radiation exposure from serial PET/CT studies performed in pediatric malignancies was considerable; however, lower doses can be used for both PET and CT studies. The ALARA principle must be applied without sacrificing diagnostic information.

Keywords PET/CT Radiation exposure

Cumulative radiation dose $\cdot$ Pediatric

\section{Introduction}

The introduction of combined PET/CT has been an important advancement in the diagnosis, staging, monitor-

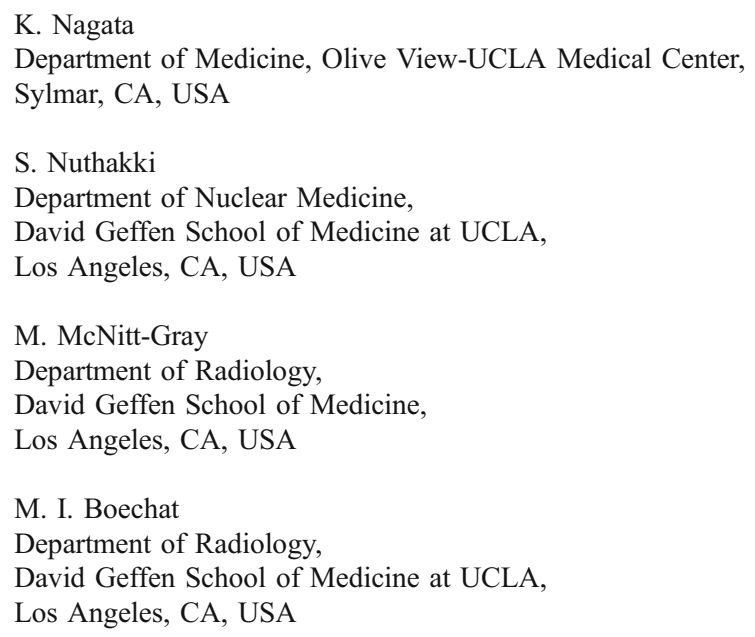


ing of response to therapy, and surveillance of various malignancies in adult patients $[1,2]$. The role of PET/CT in the management of children with malignancies is less clearly defined, although there is a growing body of literature suggesting that PET/CT is helpful in the overall management of various pediatric malignancies [3-8]. However, the increasing use of serial PET/CT scans in the management of pediatric malignancies raises the important consideration of radiation exposure in children. We estimated the cumulative radiation dose from serial PET/CT studies in children with malignancies, using our institution's standard protocol.

\section{Materials and methods}

Institutional review board approval was obtained to perform a retrospective review of children undergoing clinically indicated PET/CT studies at our institution. Two hundred forty-eight clinical PET/CT studies performed on 78 patients (50 boys/28 girls, 3 to 18.5 years of age from December 2002 to October 2007) were reviewed (Fig. 1). This review included collecting patient data including age (at the time of scan), gender, weight, clinical indication, malignancy and whether the child went on to have radiation therapy. The review also included first determining the number and dates of PET/CT as well as CT-only exams that each child underwent.

For each imaging study, image data was reviewed to determine the body region that was being examined; the start and stop location of each series was recorded for later use in estimating effective dose (ED). This was repeated for each CT and each PET performed. As part of this review, the technical parameters for each series were also recorded. For the CT scan, the following parameters were extracted from the DICOM headers: (a) scanner make and model (e.g., Siemens Emotion Duo), (b) $\mathrm{kVp}$, (c) $\mathrm{mA}$, (d) beam collimation, (e) rotation time and (f) pitch. For each PET series, the amount of FDG administered was obtained from the medical record. At our institution, both the FDG dose administered and the CT technical parameters were adjusted

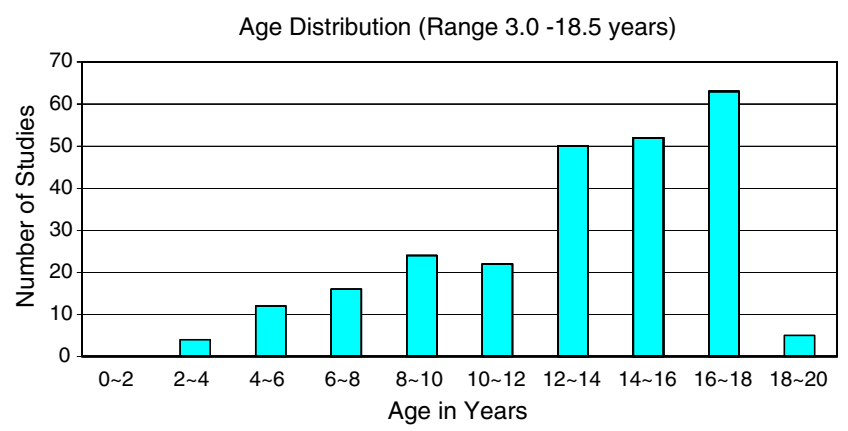

Fig. 1 Age distribution for patient weight (for FDG) and patient size (for CT), so each value had to be recorded individually. It is clinical practice in our institution to perform the $\mathrm{CT}$ scan as a diagnostic study and not just for attenuation correction; it is also our practice to use intravenous contrast agent unless there is a contraindication. In addition, it is our practice to perform a breath-hold non-contrast CT chest study in selected patients as needed to evaluate for metastatic disease in the lungs. It should also be noted that the CT scanner used in the PET/CT machine (Emotion Duo, Siemens Medical Solutions, Forcheim, Germany) does not have tube current modulation capabilities, so the $\mathrm{mA}$ value reported was the fixed $\mathrm{mA}$ value used in the study.

Effective dose from each CT series was estimated using the ImPACT Patient Dosimetry Calculator (www.impactscan.org) (Version 0.99x) with patient-specific scan parameters. This software package makes use of the National Radiation Protection Board (NRPB) data sets produced in report SR250, which provides normalized organ dose data for irradiation of a mathematical phantom (MIRD phantom) by a range of $\mathrm{CT}$ scanners obtained through Monte Carlo simulations $[9,10]$. The specific parameters obtained from the DICOM file headers are used as inputs to the software, as are start and stop locations of the series, which users can interactively select using a diagram of the MIRD phantom provided. Based on these inputs, the software estimates the radiation dose to each organ and then calculates the estimated whole-body ED in $\mathrm{mSv}$. This was recorded for each CT series for each patient.

The estimate provided by the ImPACT Dosimetry Calculator is for a standard adult model, so the ED from CT was adjusted according to age. This adjustment was performed based on results reported by Khursheed et al. [11], who performed a series of Monte Carlo calculations on a range of phantoms corresponding to newborn, 1-year-old, 5-year-old, 10-year-old, 15-year-old and adult patients [11]. From these results, a table of effective-dose adjustment factors was created based on an interpolation among the results obtained for these ages. This is shown in Table 1 .

Table 1 Factors used to adjust effective dose from CT by patient age; derived from Khursheed et al [11]

\begin{tabular}{lccc}
\hline Age in years & Factor & Age in years & Factor \\
\hline 3 & 1.60 & 11 & 1.26 \\
4 & 1.55 & 12 & 1.22 \\
5 & 1.50 & 13 & 1.18 \\
6 & 1.46 & 14 & 1.14 \\
7 & 1.42 & 15 & 1.10 \\
8 & 1.38 & 16 & 1.07 \\
9 & 1.34 & 17 & 1.03 \\
10 & 1.30 & 18 & 1.00 \\
\hline
\end{tabular}


Effective dose from each PET series was estimated using the OLINDA software (Version 1.0, Vanderbilt University, Nashville, TN, USA) with patient-specific FDG doses. OLINDA is a program used to calculate internal radiation dose estimates for radionuclides used in nuclear medicine. The program has phantom libraries that permit the calculation of doses for individuals of different ages and sizes [12]. Nuclide and kinetic models need to be provided by the users. F-18, which is the essential nuclide in FDG, was specified. An absorbed dose estimation study from F-18 FDG by Hays et al. [13] was used as a kinetic model. Adult male, adult female, 15-year, 10-year, 5-year, 1-year models in OLINDA's phantom library were utilized to generate effective dose conversion factor $(\mathrm{mSv} / \mathrm{mCi})$. These factors were then also interpolated according to different ages, shown in Table 2, and then multiplied by injected activity $(\mathrm{mCi})$ for each PET study to get an estimation of effective dose.

\section{Results}

The average age of the children in this study was 13.1 years with a range from 3.0 to 18.5 years. There were 10 children in the 0 - to 5 -year-old range, 46 in the 5- to 10 -year-old range, 98 in the 10- to 15 -year-old range and 94 in the 15 to 18 -year-old range at the time of the study. The weights of the children (at time of scan) ranged from 30 to 230 pounds, with an average of 112 pounds.

The typical parameters for the CT study was: $130 \mathrm{kVp}$ (79.4\%) and $110 \mathrm{kVp}(20.6 \%), \mathrm{mA}$ range from 43 to 170 with an average of 111.3, rotation time of either $0.8 \mathrm{~s}$ or $1.0 \mathrm{~s}$, pitch range from 1.0 to 1.3 and the vast majority had a beam collimation of $2 \times 5 \mathrm{~mm}$. The majority of these scans were from the mid-brain to mid-thigh. For the PET portion, $0.21 \mathrm{mCi} / \mathrm{kg}$ of FDG was given on a straight weight basis.

The average number of PET/CT studies was 3.2 per patient (range: 1-14) (Fig. 2). The average effective dose of an

Table 2 Effective dose conversion factors from PET for both male and female pediatric patients as a function of age

\begin{tabular}{|c|c|c|c|c|c|}
\hline \multirow[t]{2}{*}{ Age in years } & \multicolumn{2}{|c|}{$\begin{array}{l}\text { Effective dose } \\
(\mathrm{mSv} / \mathrm{mCi})\end{array}$} & \multirow[t]{2}{*}{ Age in years } & \multicolumn{2}{|c|}{$\begin{array}{l}\text { Effective dose } \\
(\mathrm{mSv} / \mathrm{mCi})\end{array}$} \\
\hline & Male & Female & & Male & Female \\
\hline 3 & 1.12 & 1.12 & 11 & 0.49 & 0.50 \\
\hline 4 & 0.95 & 0.95 & 12 & 0.45 & 0.48 \\
\hline 5 & 0.78 & 0.78 & 13 & 0.42 & 0.46 \\
\hline 6 & 0.73 & 0.73 & 14 & 0.39 & 0.44 \\
\hline 7 & 0.68 & 0.68 & 15 & 0.35 & 0.42 \\
\hline 8 & 0.62 & 0.62 & 16 & 0.33 & 0.40 \\
\hline 9 & 0.57 & 0.57 & 17 & 0.30 & 0.38 \\
\hline 10 & 0.52 & 0.52 & 18 & 0.27 & 0.36 \\
\hline
\end{tabular}

Number of studies per patient

(Average 3.2 studies/pt, Range:1-14)

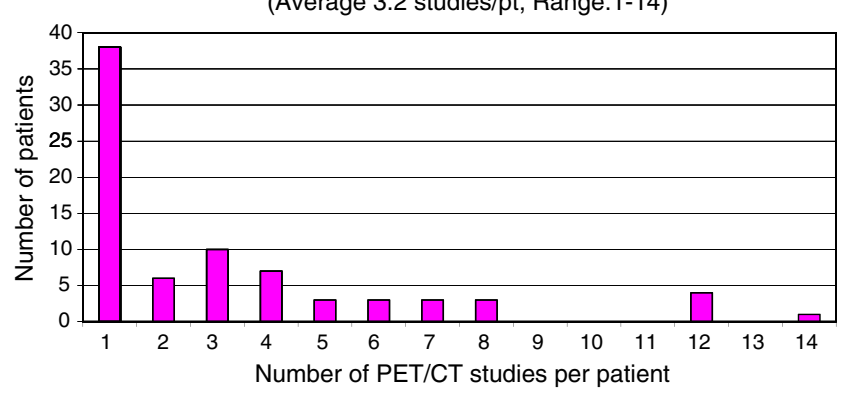

Fig. 2 Study distribution

individual CT study was $20.3 \mathrm{mSv}$ (range: 2.7-54.2) (Fig. 3), of PET study was $4.6 \mathrm{mSv}$ (range: 0.4-7.7) (Fig. 4) and of PET/CT study was $24.8 \mathrm{mSv}$ (range: 6.2-60.7) (Fig. 5).

The average cumulative dose per patient from CT studies was $64.4 \mathrm{mSv}$ (range: 2.7-326) (Fig. 6), from PET studies was $14.5 \mathrm{mSv}$ (range: 2.8-73) (Fig. 7) and from PET/CT studies was $78.9 \mathrm{mSv}$ (range: 6.2-399) (Fig. 8).

Radiation doses varied significantly depending on the number of studies as well as the number of additional CT scans performed. A total of 199 chest CT scans were performed to evaluate the metastatic disease in the lungs. The average effective dose of an individual chest CT study was $2.8 \mathrm{mSv}$ (range: 0.43-7.9). Fifty-eight percent (45 children) received no radiation therapy and $42 \%$ (33 patients) received radiation therapy. Twenty-seven percent (21 children) of all patients received $>100 \mathrm{mSv}$ cumulative dose; this consisted of $9 \%$ ( 7 children) with no radiation therapy, and $18 \%$ (14 children) with radiation therapy. Figures 9 and 10 show the effective dose distribution from an individual PET/CT and cumulative PET/CT as function of age groups ( $0-5$ years, $5-10$ years and $>10$ years).

\section{Discussion}

The radiation exposure from PET/CT studies is considerable, especially for those children undergoing regular

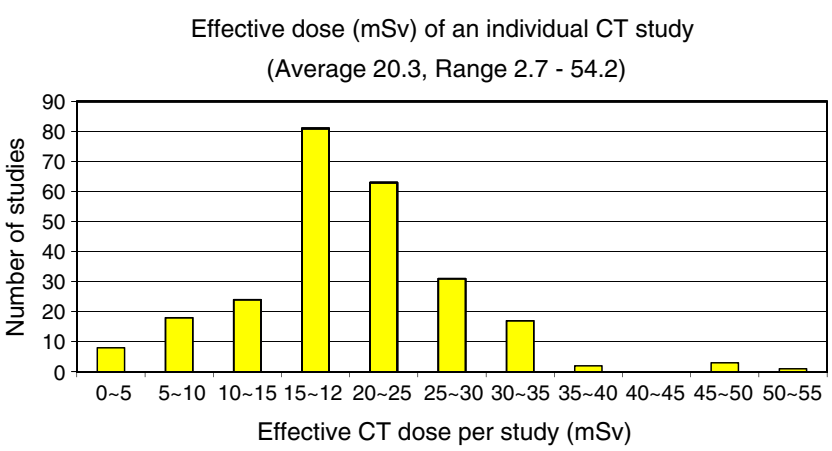

Fig. 3 Effective dose (mSv) of an individual CT study 
Effective dose (mSv) of an individual PET study (Average 4.6. Range: 0.4 - 7.7)

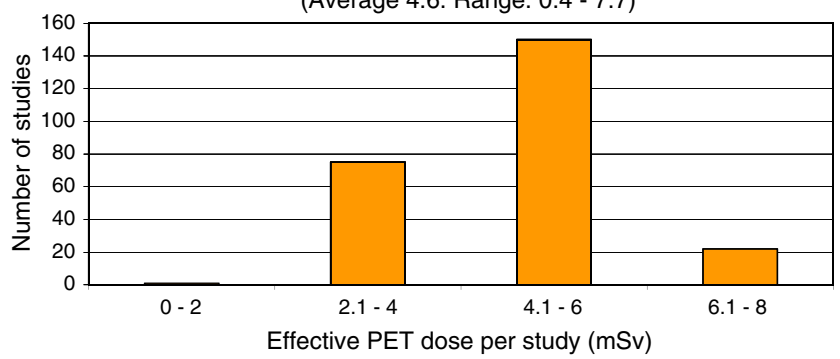

Fig. 4 Effective dose (mSv) of an individual PET study

follow-up exams. Radiation doses vary significantly depending on the number of PET/CT studies performed. The results from this study demonstrate that the largest portion of the radiation dose comes from the $\mathrm{CT}$ portion of the examination. The effective doses from the CT studies ranged from 2.7 to $54.2 \mathrm{mSv}$ and nearly half of the studies exceeded $20 \mathrm{mSv}$. These studies all involved "full-body" CT scans, which ranged from mid-brain to mid-thigh and thus irradiated essentially all of the radiosensitive organs. The techniques for these scans were usually adjusted for patient size with $\mathrm{kVp}$ and/or $\mathrm{mAs}$ being reduced for smaller/younger patients. For those studies exceeding $20 \mathrm{mSv}$, a typical set of technical parameters was $130 \mathrm{kVp}, 100$ to $130 \mathrm{~mA}, 1$-s rotation time, $2 \times 5-\mathrm{mm}$ beam collimation and pitch 1 . After performing the age adjustment described above, this would often result in effective doses that exceeded $20 \mathrm{mSv}$. This effective dose is actually reasonably consistent with the effective doses received by adults undergoing similar anatomic coverage, even though the technical factors (e.g., $\mathrm{mA}$ ) would be increased due to the increased size of the adult patient. It should also be noted that the average age of our patient population was 13.1 years at the time of the study, that $77 \%$ of our patient population was $>10$ years old and that average weights were 112 pounds with $64 \%$ exceeding 100 pounds.

The long-term effects of the treatment of pediatric malignancies are numerous and substantial. These include development of cardiomyopathy, avascular necrosis of the hip, cognitive delay, early onset of heart disease, pulmonary

Effective dose (mSv) of an individual PET/CT study (Average 24.8, Range 6.2 - 60.7)

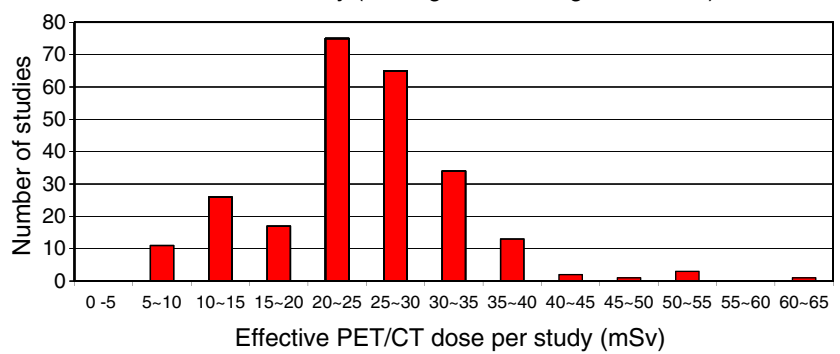

Fig. 5 Effective dose $(\mathrm{mSv})$ of an individual PET/CT study

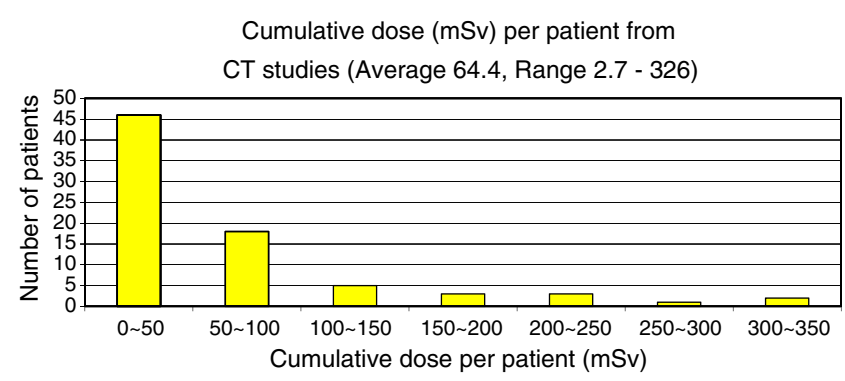

Fig. 6 Cumulative dose (mSv) per patient from CT studies

fibrosis and increased risk of secondary malignancies. Children who receive alkylating chemotherapeutic agents and radiation as part of their treatment regimens are at particular risk for developing secondary malignancies. The increasing use of PET/CT in the management of pediatric malignancies raises the important issue of radiation exposure in children, particularly when it is reported that children have an increased risk of developing secondary malignancies from radiation exposure compared with adults. In fact, by extrapolating data from atomic bomb survivors, this increased risk is by an order of magnitude greater than that of adults [14-16]. It has been estimated, but not proved, that the lifetime cancer risk of a 1-year-old who has received an abdominal CT is 1 in 550 [17]. At doses of $100 \mathrm{mSv}$ or greater ( $21 \%$ of the patients reported), the cancer risk has been estimated at 1 in 100 individuals [18]. Judicious use of imaging is fundamental for the management of these patients, using tailored protocols that follow the ALARA principle (as low as reasonably achievable) to obtain diagnostic information.

The significance of incremental radiation exposure from serial PET/CT scans for children who have received highdose radiation therapy as part of their treatment is not known. The significance of the incremental exposure may also depend on the location of radiation; for example, one could compare patients who receive mantle radiation versus limited radiation to a distal extremity. We suggest that the cumulative radiation dose to organs such as the lung, heart, thyroid, gonads, etc., is significant.

Cumulative dose $(\mathrm{mSv})$ per patient from

PET studies (Average 14.5, Range: 2.8 - 73)

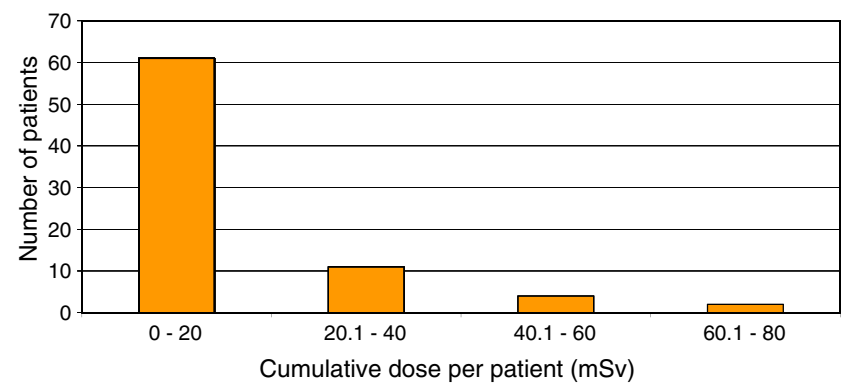

Fig. 7 Cumulative dose (mSv) per patient from PET studies 


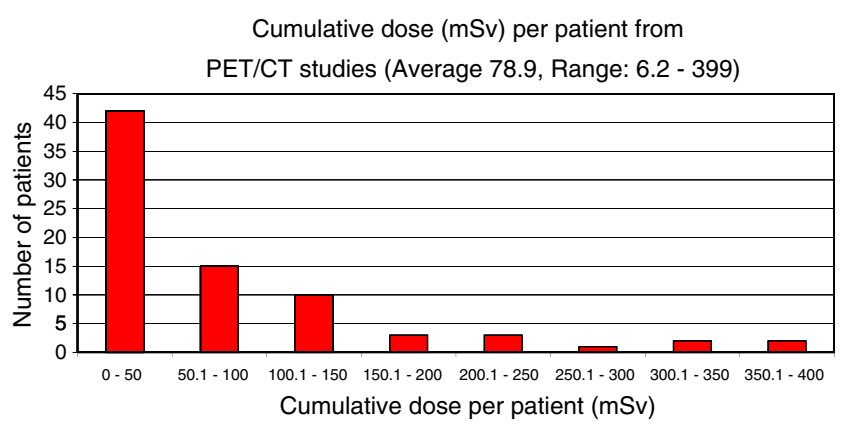

Fig. 8 Cumulative dose $(\mathrm{mSv})$ per patient from PET/CT studies

We have estimated the dose of radiation from serial PET/ CT scans in pediatric patients, but it is important to note that the cumulative radiation from other imaging modalities has not been included. These imaging modalities include the technetium 99 bone scans, ${ }^{123}$ Iodine MIBG (metaiodobenzylguanidine) scans, interval CT studies for acute events and fluoroscopy, which also add to the radiation dose.

It is necessary to apply the ALARA principle in the radiation exposure of PET/CT without sacrificing diagnostic information. The greatest contributor to overall radiation exposure in PET/CT is the whole-body diagnostic CT. It is widely accepted that by decreasing the tube current $(\mathrm{mA})$ value on the $\mathrm{CT}$ portion the radiation dose can be reduced substantially. It is clear that until the standards of frequency, interval and number of needed PET/CT scans is established in the management of pediatric malignancies, that the use of PET/CT in children should be used judiciously on a case-by-case basis with particular emphasis on the risk, benefit and cumulative radiation dose to children. We have reviewed the CT protocols for all pediatric studies at our institution and decreased doses following "image gently" guidelines, according to patient weight. Doses of FDG for PET have also been reduced to $0.14 \mathrm{mCi} / \mathrm{Kg}$, as recommended in the literature. We are now monitoring all pediatric CTs and PET/CT studies for dose (Table 3).

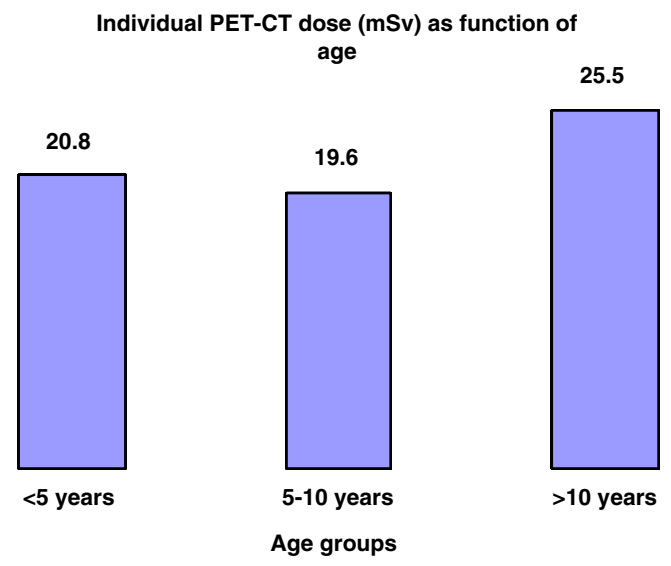

Fig. 9 Individual PET-CT dose (mSv) as function of age

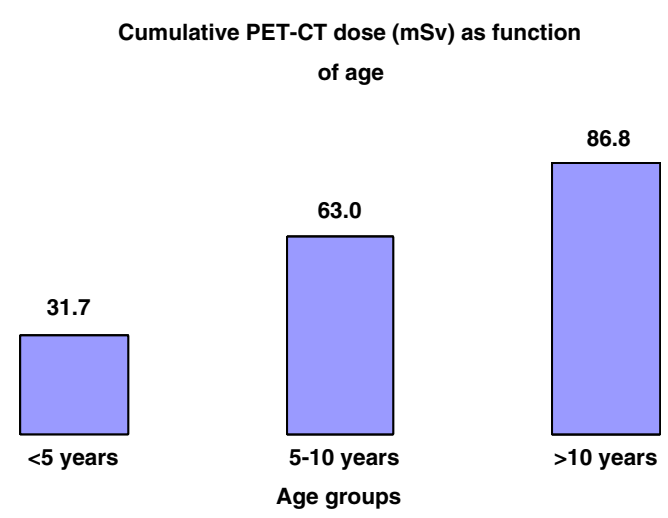

Fig. 10 Cumulative PET-CT dose (mSv) as function of age

Alternative approaches to the use of whole-body PET/ CT include routine CT or PET/CT limited to the area of interest, intercalated by periodic whole-body PET/CT, and whole-body PET followed by limited CT in areas of PETpositive lesions (albeit with some reduced sensitivity).

Whole-body PET/CT continues to be an important noninvasive diagnostic/staging modality for certain malignancies such as Hodgkin and non-Hodgkin lymphomas, and it is thought that alternative or more cautious approaches should be weighed against the unequivocal benefit provided. In order to reduce the radiation exposure from imaging studies in children with cancer we suggest the following guidelines:

1. Strict adherence to the ALARA principle. This may involve decreasing the CT tube $(\mathrm{mA})$ current and avoidance of whole-body scans when only a limited scan is sufficient.

2. Use of pediatric protocols and, when possible, imaging at pediatric centers where radiologists and technicians are acutely aware of the need to reduce radiation exposure.

3. Establishment of formal guidelines for the interval, number, and frequency of PET/CT scans for each pediatric malignancy.

4. When appropriate, use of modalities such as MR or US to reduce radiation exposure.

5. Overall attention to the cumulative radiation exposure for each child.

Table 3 Current parameters for CT chest and abdomen at our institution

\begin{tabular}{lrrrr}
\hline & KV & mAs & KV & mAs \\
\hline$<5 \mathrm{~kg}$ & 80 & 45 & 80 & 45 \\
$6-15 \mathrm{~kg}$ & 80 & 55 & 80 & 55 \\
$16-60 \mathrm{~kg}$ & 100 & 55 & 100 & 65 \\
$>61 \mathrm{~kg}$ & 120 & 55 & 120 & 65 \\
\hline
\end{tabular}




\section{Conclusion}

The radiation exposure from serial PET/CT studies performed in pediatric malignancies can be considerable. The ALARA principle must be applied without sacrificing diagnostic information either by reducing $\mathrm{CT}$ tube current or by considering alternative diagnostic approaches such as limited CT scan length or non-radiating modalities like MR and US.

PET/CT continues to be an important noninvasive diagnostic, staging, and surveillance modality for certain pediatric malignancies. The decision to utilize PET/CT in children should be made on an individual basis with particular awareness to the cumulative radiation exposure and the overall benefit of the scan.

Acknowledgement A portion of this work was supported by a grant from the National Institute of Biomedical Imaging and Bioengineering (NIBIB) PHS \# R01EB004898.

Open Access This article is distributed under the terms of the Creative Commons Attribution Noncommercial License, which permits any noncommercial use, distribution, and reproduction in any medium, provided the original author(s) and source are credited.

\section{References}

1. Czernin J, Allen-Auerbach M, Schelbert HR (2007) Improvements in cancer staging with PET/CT: literature-based evidence as of September 2006. J Nucl Med 48:78S-88S

2. Czernin J, Schelbert HR (2004) PET/CT imaging: facts, opinions, hopes, and questions. J Nucl Med 45:1S-3S

3. Federman N, Feig SA (2007) PET/CT in the evaluating pediatric malignancies: a clinician's perspective. J Nucl Med 48:1920-1922
4. Tatsumi M, Miller JH, Wahl RL (2007) FDG PET/CT in evaluating pediatric malignancies. J Nucl Med 48:1923-1931

5. Rhodes MM, Delbeke D, Whitlock JA et al (2006) Utility of FDG-PET/CT in follow-up of children treated for Hodgkin and non-Hodgkin lymphoma. J Pediatr Hematol Oncol 28:300-306

6. Miller E, Meltser U, Avrahami G et al (2006) Role of 18F-FDG $\mathrm{PET} / \mathrm{CT}$ in staging and follow-up of lymphoma in pediatric and young adult patients. J Comput Assist Tomogr 30:689-694

7. Hudson MM, Donaldson SS (1999) Treatment of pediatric Hodkin's lymphoma. Semin Hematol 36:313-323

8. McCarville MB, Christie R, Daw NC (2005) PET/CT in the evaluation of childhood sarcomas. AJR 184:1293-1304

9. Jones DG, Wall BF (1985) Organ doses from medical x-ray examinations calculated using Monte Carlo techniques. National Radiological Protection Board Report NRPB-R186

10. Jones DG, Shrimpton PC (1991) Survey of CT practice in the UK part 3: normalized organ doses calculated using Monte Carlo techniques. National Radiological Protection Board Report NRPB-R250

11. Khursheed A, Hillier MC, Shrimpton PC et al (2002) Influence of patient age on normalized effective doses calculated for CT examinations. Br J Radiol 75:819-830

12. Stabin MG, Siegel JA (2003) Physical models and dose factors for use in internal dose assessment. Health Phys 85:294-310

13. Hays MT, Watson EE, Thomas SR et al (2002) MIRD dose estimate report No. 19: radiation absorbed dose estimates from ${ }^{18}$ F-FDG. J Nucl Med 43:210-214

14. Otsuka H, Graham MM, Kubo A et al (2005) FDG-PET/CT findings of sarcomatous transformation in neurofibromatosis: a case report. Ann Nucl Med 19:55-58

15. Pierce DA, Shimizu Y, Preston DL et al (1996) Studies of the mortality of atomic bomb survivors. Report 12, part 1. Cancer: 1950-1990. Radiat Res 146:1-27

16. Brenner DJ, Hall EJ (2007) Computed tomography-an increasing source of radiation exposure. N Engl J Med 357:2277-2284

17. Brenner DJ, Elliston CD, Hall EJ et al (2002) Estimated risks of radiation-induced fatal cancer from pediatric CT. AJR 176:289-296

18. Kleinerman RA (2006) Cancer risks following diagnostic and therapeutic radiation exposure in children. Pediatr Radiol 36 (Suppl 2):121-125 